EPJ Web of Conferences 102, 00003 (2015)

DOI: $10.1051 /$ epjconf/ 201510200003

C) Owned by the authors, published by EDP Sciences, 2015

\title{
Dust in protoplanetary disks: observations ${ }^{\star}$
}

\author{
L.B.F.M. Waters ${ }^{1,2}$ \\ ${ }^{1}$ SRON Netherlands Institute for Space Research, Sorbonnelaan 2, 3584 CA Utrecht, The Netherlands \\ ${ }^{2}$ Anton Pannekoek Institute for Astronomy, University of Amsterdam, PO BOX 94249, 1090 GE Amsterdam, \\ The Netherlands
}

\begin{abstract}
Solid particles, usually referred to as dust, are a crucial component of interstellar matter and of planet forming disks surrounding young stars. Despite the relatively small mass fraction of $\approx 1 \%$ (in the solar neighborhood of our galaxy; this number may differ substantially in other galaxies) that interstellar grains represent of the total mass budget of interstellar matter, dust grains play an important role in the physics and chemistry of interstellar matter. This is because of the opacity dust grains at short (optical, UV) wavelengths, and the surface they provide for chemical reactions. In addition, dust grains play a pivotal role in the planet formation process: in the core accretion model of planet formation, the growth of dust grains from the microscopic size range to large, $\mathrm{cm}$-sized or larger grains is the first step in planet formation. Not only the grain size distribution is affected by planet formation. Chemical and physical processes alter the structure and chemical composition of dust grains as they enter the protoplanetary disk and move closer to the forming star. Therefore, a lot can be learned about the way stars and planets are formed by observations of dust in protoplanetary disks. Ideally, one would like to measure the dust mass, the grain size distribution, grain structure (porosity, fluffiness), the chemical composition, and all of these as a function of position in the disk. Fortunately, several observational diagnostics are available to derive constrains on these quantities. In combination with rapidly increasing quality of the data (spatial and spectral resolution), a lot of progress has been made in our understanding of dust evolution in protoplanetary disks. An excellent review of dust evolution in protoplanetary disks can be found in Testi et al. (2014).
\end{abstract}

\section{Scattering, absorption and thermal emission of dust}

The interaction of radiation with dust particles in the interstellar medium (ISM) is beautifully illustrated by the optical and infrared images of the famous Horsehead nebula in Orion (M33), see Fig. 1. The dense molecular cloud absorbs light from behind the cloud. Bright stars illuminate the cloud, causing a reflection nebula. At longer, infrared wavelengths, the thermal emission from the dust grains, heated by the diffuse interstellar radiation field and nearby young hot stars, begins to dominate.

Due to the steep wavelength dependence of scattering opacity $\left(\kappa_{v}^{\text {sca }} \propto \lambda^{-4}\right.$ for small grains in the so-called Rayleigh limit), scattering dominates at the shortest wavelengths. Often the intensity of

$\star 2^{\text {nd }}$ Lecture of the Summer School "Protoplanetary Disks: Theory and Modelling Meet Observations"

This is an Open Access article distributed under the terms of the Creative Commons Attribution License 4.0, which permits unrestricted use, distribution, and reproduction in any medium, provided the original work is properly cited. 

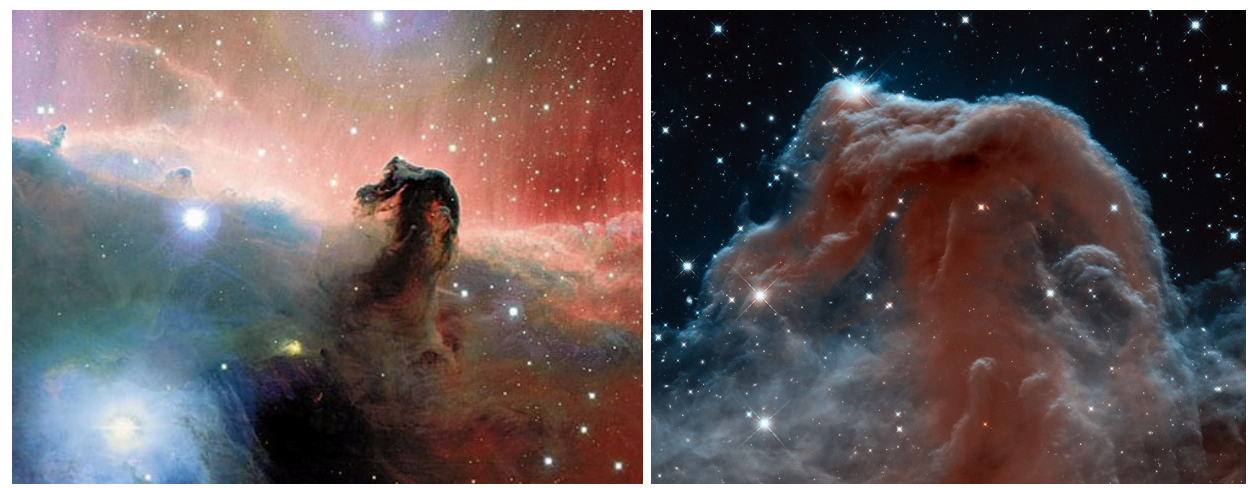

Figure 1. Optical and infrared images of the Horsehead Nebula, showing absorption, scattering and emission from interstellar dust. Image Credits: ESO/J. Emerson/VISTA. Acknowledgment: Cambridge Astronomical Survey Unit Credit for Hubble Data: HLA, Hubble Heritage Team (STScI/AURA) Composite Assembly and Processing: Robert Gendler (http://www.robgendlerastropics.com).

scattered light detected in reflection nebulae has a steeper wavelength dependence (is bluer) than that of the source irradiating the dust particles. Absorption $\left(\kappa_{v}^{\mathrm{abs}} \propto \lambda^{-1}\right.$ for small grains in the Rayleigh limit) dominates the dust opacity at longer wavelengths. The densest dark clouds are even seen in absorption at mid-infrared wavelengths, where they absorb radiation from warm dust grains behind these clouds.

The wavelength dependence of the dust opacity is a strong function of the properties of the dust grains, most importantly their size and chemical composition. Often the size parameter $x$ is used to distinguish between large and small grains compared to the wavelength: $x=\frac{2 \pi a}{\lambda}$, where $a$ is the grain radius and $\lambda$ is the wavelength. In the Rayleigh limit $x \ll 1$, reached at long wavelengths compared to the size of the particle. For large $x$, surface effects dominate the interaction between the electromagnetic wave and the particle, and the opacity saturates to a value proportional to the geometric cross-section $\pi a^{2}$, and becomes wavelength independent. Figure 3 illustrates the effect of grain size on the opacity of amorphous silicate grains of 0.1 and $3.5 \mu \mathrm{m}$ as a function of wavelength. The $0.1 \mu \mathrm{m}$ grain shows a continuously rising opacity towards shorter wavelength, while the $3.5 \mu \mathrm{m}$ has an essentially constant opacity for wavelengths short wards of a few $\mu \mathrm{m}$.

At infrared (IR) wavelengths the thermal emission from dust dominates. The temperature of a dust grain is determined by the balance between the amount of energy absorbed, and the radiation it emits, both per unit time. Whether a grain has a constant, well defined temperature or shows temperature fluctuations depends on the heat capacity of the grain and on the energy density of the radiation field in which the grain is embedded. In the limit of very small grains (or large molecules), the surface to mass ratio is large and the absorption of a single optical or UV photon can significantly increase the temperature of the particle. By emitting (near-IR) photons the temperature of the grain decreases rapidly again: if the cooling timescale of the grains is short compared to the heating timescale set by the absorption of the next photon, temperature fluctuations are present. For larger particles (typically larger than an few tens of nanometers) the surface to mass ratio is small and the absorption of a single photon only marginally changes the total energy content of the particle, resulting in a constant temperature.

A population of very small grains in the diffuse ISM will therefore show a (much) higher average temperature (typically several 100 to $1000 \mathrm{~K}$ ) than that expected for large, macroscopic grains (typically 10-20 K). Indeed, interstellar dust clouds show prominent emission at near/mid-IR wave- 


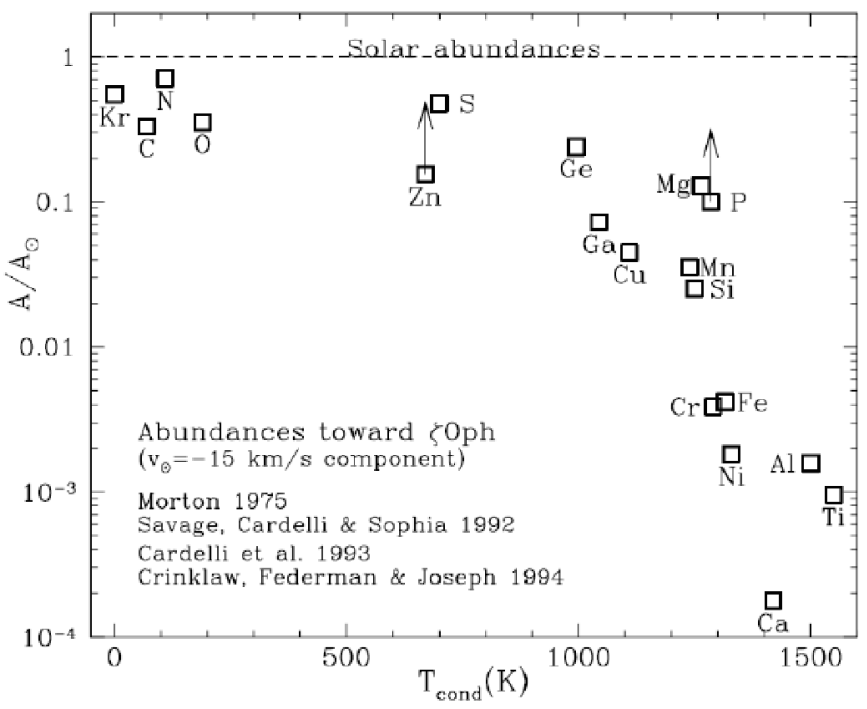

Figure 2. Depletion of interstellar gas-phase elements towards $\zeta$ Oph (plotted as the abundance A normalized to the solar abundance $\mathrm{A}_{\odot}$ ) as a function of their condensation temperature (in $\mathrm{K}$ ). Figure reprinted with permission from (Draine 2004).

lengths $(3-60 \mu \mathrm{m})$, due to the presence of super-heated very small grains and Polycyclic Aromatic Hydrocarbon (PAH) molecules: these are ubiquitous in interstellar space, and in protoplanetary disks.

\section{Dust composition}

There are two important means to study the chemical composition of dust: depletion of gas-phase elements that are locked up in dust, and spectroscopy of dust both at X-rays and in the thermal infrared. The gas-phase depletion method works in the ISM, by measuring the line strengths of interstellar absorption lines of atoms or ions detected through lines of sight towards bright background sources (usually hot stars). These line strengths can be converted to column densities, and corrected for the ionization fraction of elements in the ISM (these correction factors can be large if the observed ionization stage is not the dominant one). After normalization to the hydrogen column density the abundances can be compared to e.g. the solar abundance, and a depletion fraction derived. ISM gasphase depletions are shown in Fig. 2. A clear pattern emerges that highly refractory elements (with a high condensation temperature) are strongly depleted from the ISM, while more volatile elements are less depleted. The interpretation is that it is more difficult to destroy highly refractory dust species in the ISM through shocks.

$\mathrm{X}$-ray absorption spectroscopy (roughly in the $1-10 \mathrm{keV}$ energy domain) is a powerful tool to study the composition of interstellar gas and dust. This is because X-rays can trace both the gas-phase and solid component of elements (for grains smaller than $\sim 10 \mu \mathrm{m}$ ). Electrons in the inner shells (e.g. 1s, 1p) can absorb an X-ray photon, resulting in a bound-free transition. When in a solid, the emitted photo-electron will interact with the nearest atoms, causing a slight modulation in the X-ray absorption cross-section near the ionization edge. High resolution spectroscopy can distinguish the lattice structure of the solid material and thus provide information about the chemical composition of the dust grains, e.g. whether $\mathrm{Fe}$ is predominantly in the form of metallic $\mathrm{Fe}$ or in an oxide (Costantini 


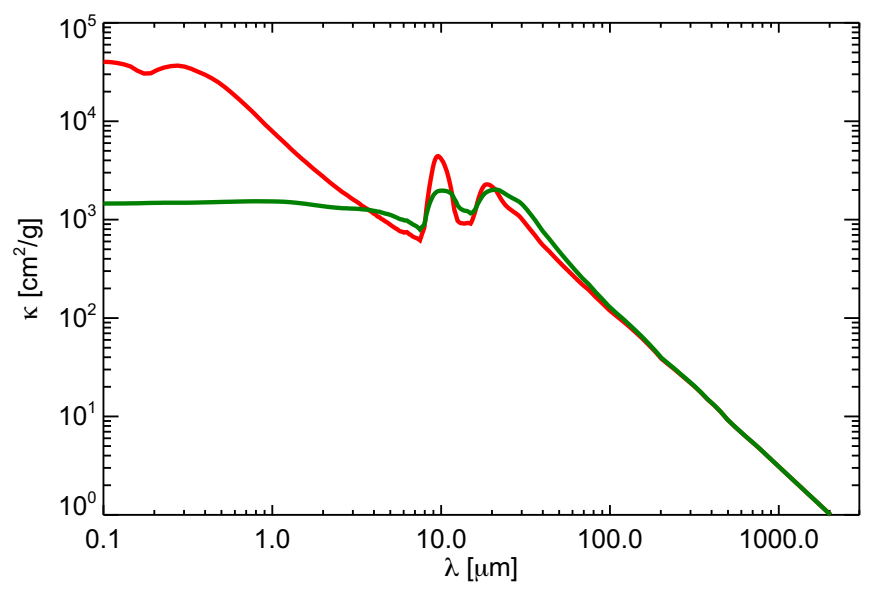

Figure 3. Opacities of amorphous silicate grains of 0.1 and $3.5 \mu \mathrm{m}$ size. The $3.5 \mu \mathrm{m}$ shows a much lower, and roughly wavelength independent behavior at short wavelengths, while the opacity of the $0.1 \mu \mathrm{m}$ grain continues to increase to short wavelengths. Notice also the weaker 10 and $18 \mu \mathrm{m}$ silicate opacity peaks in the larger of the two grains. Figure from M. Min, private communication.

et al. 2014; Lee \& Ravel 2005). In our galaxy, accreting compact objects are used as bright background $\mathrm{X}$-ray sources. These studies have revealed evidence that silicates in the diffuse ISM are Mg-rich, with $\mathrm{Fe}$ in the form of metallic Fe (Costantini et al. 2012).

At mid-infrared wavelengths, strong vibrational resonances of abundant dust species are detected both in absorption (bright background stars detected through cold molecular cloud dust), and in emission (circumstellar dust ejected by red giants, or dust in protoplanetary disks and debris disks). These resonances are due to stretching and bending modes of molecules in the lattice, and are therefore probes of both the chemical composition of the dust as well as the lattice structure (amorphous versus crystalline). The shape and strength of the resonance also depends on grain size and grain shape, making a reliable identification of the detected dust species sometimes difficult. Identifications are more robust when more than one resonance can de identified, such as forsterite $\left(\mathrm{Mg}_{2} \mathrm{SiO}_{4}\right)$. With increasing size grains can become opaque at the wavelength of the resonance, weakening it (not all molecules in the grain contribute to the opacity at that wavelength) and eventually the resonance is no longer detectable. Therefore mid-IR spectroscopy is only sensitive to grain composition for grain sizes less than a few microns. In Fig. 3 the effect of grain size on the shape and strength of amorphous silicate opacities is shown. Table 1 lists some of the most common oxygen-rich dust species with their IR resonances.

\section{Dust in protoplanetary disks}

\subsection{Processing of dust in disks}

The process of star- and planet formation involves huge changes in densities and temperatures, as material from the (diffuse) ISM enters a molecular cloud and subsequently becomes part of a young star and its environment. These changes affect the size, morphology and chemical composition of the dust. In the diffuse ISM, dust consists predominantly of amorphous silicates (about $70 \%$ by mass) and amorphous carbon or graphitic material (30\% by mass). The shape of the interstellar extinction curve can be used to derive constrains on grain size in the ISM, that are typically between 0.01 and 
about $0.5 \mu \mathrm{m}$. Grains grow due to the slow accretion of atoms and, in the molecular cloud, by the accretion of molecules that form a volatile ice layer. In addition, grains collide and stick, resulting in the formation of chemically inhomogeneous, fluffy dust aggregates. In protoplanetary disks, grains settle to the mid-plane and as a result grow, compact, and drift inwards due to the slightly smaller orbital velocity of the gas compared to the dust.

Near the star, temperatures rise causing the volatile ices to evaporate to the gas phase, releasing new complex (organic) molecules that have formed in the ice. In the inner disk, temperatures can be high enough to change the chemical composition and lattice structure of the dust, or even cause the evaporation and re-condensation of dust. For instance, the glass temperature of silicates is about $1000 \mathrm{~K}$, above which the amorphous lattice structure changes into the energetically more favorable crystalline lattice structure. Heating also causes Fe to diffuse out of the silicate lattice, resulting in the formation of $\mathrm{Mg}$-rich silicates and metallic Fe. At the highest temperatures and pressures, only the most refractory materials can survive, such as $\mathrm{Al}_{2} \mathrm{O}_{3}$. A small fraction of these high temperature solids produced in the inner disk are transported outwards by radial mixing. Planet formation and planet migration clear parts of the disk, causing the formation of large gaps and/or holes. The spatial distribution of dust in disks can therefore provide a wealth of information about the physical and chemical processes related to planet formation.

\subsection{Spectral Energy Distribution}

Thermal emission of dust in the disk is detected at long, infrared and millimeter wavelengths, where it dominates the spectral energy distribution (SED). The temperature distribution of dust in disks is a complex function of the radiation field, the disk geometry, and of the opacity and volume density of dust particles. In a few simplified limiting cases, expressions can be derived for the temperature dependence of dust as a function of distance from the star. It can be shown that, assuming optically thin dust at all wavelengths, and assuming a dust opacity $\kappa_{v}^{\text {abs }}$ (units $\mathrm{cm}^{2} / \mathrm{g}$ ) proportional to $v^{p}$, that the temperature distribution of grains as a function of distance is $T(r) \propto r^{-2 /(4+p)}$. For black body grains (i.e. grains that absorb and emit as black bodies), $p=0$ and so we get the familiar result $T(r) \propto r^{-1 / 2}$. Adopting a dust density gradient $n(r) \propto r^{-m}$, the spectral slope of the dust SED can be derived:

$$
F_{v} \propto v^{3+p-0.5(3-m)(4+p)}
$$

These optically thin temperature gradients of course do not apply to the disk mid-plane, but give a first order estimate of the temperatures in the optically thin disk surface layers, that dominate the SED in the $10-100 \mu \mathrm{m}$ wavelength range (see below). Disks reprocess stellar light, by absorbing stellar

Table 1. Common oxygen-rich circumstellar dust species in protoplanetary disks

\begin{tabular}{|l|cc|}
\hline$\lambda(\mu \mathrm{m})$ & identification & lattice structure \\
\hline 9.7 & $\begin{array}{c}\text { Si-O stretch } \\
\text { in silicates } \\
\text { O-Si-O bend } \\
18\end{array}$ & amorphous \\
& in silicates & amorphous \\
$11.3,16.5,19.5$, & Forsterite $\left(\mathrm{Mg}_{2} \mathrm{SiO}_{4}\right)$ & crystalline olivine \\
$23.5,27.5,33.6,69$ & Enstatite ${ }^{1}\left(\mathrm{MgSiO}_{3}\right)$ & crystalline pyroxene \\
$9.3,40.5,43$ & $\mathrm{SiO}_{2}$ & crystalline \\
9,21 & $\mathrm{H}_{2} \mathrm{O}$ ice & crystalline \\
44,60 & &
\end{tabular}

${ }^{1}$ Enstatite shows several more, weaker resonances in the $20-40 \mu \mathrm{m}$ spectral range. 


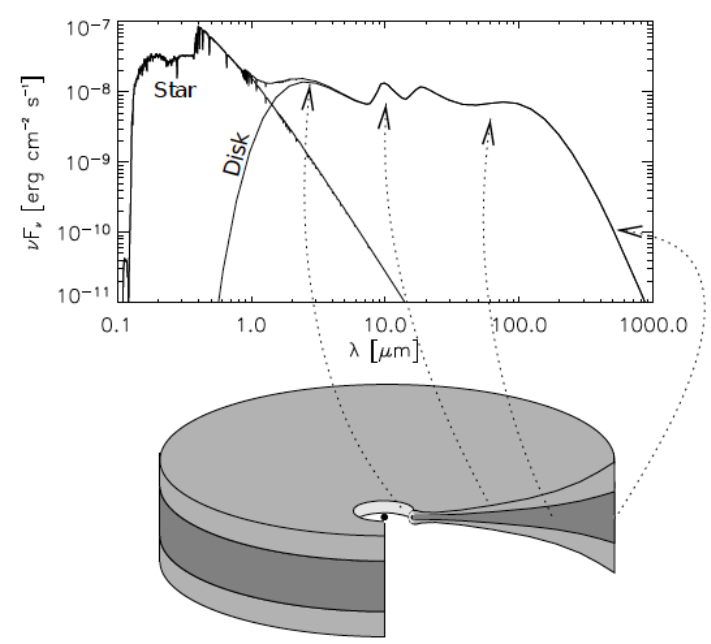

Figure 4. Schematic spectral energy distribution of a protoplanetary disk. The optical spectrum is dominated by the stellar photosphere, while at IR and longer wavelengths the disk dominates. Shown is the region from which different parts of the disk spectrum are emitted. Taken with permission from Mulders, thesis, University of Amsterdam, adopted from (Dullemond et al. 2007, from Protostars and Planets V by Reipurth et al. (c) 2007, the Arizona Board of Regents, reprinted by permission of the University of Arizona Press).

optical/UV photons and re-emitting that energy in the infrared. The reprocessing efficiency of disks is rather high: depending on the inclination angle under which the disk is viewed, optically thick passive disks can reprocess up to $\sim 50 \%$ of the stellar luminosity (accretion luminosity is ignored here).

Usually three different wavelength regimes are distinguished (Fig. 4), corresponding to different physical regions in the disk: $1-10 \mu \mathrm{m}, 10-100 \mu \mathrm{m}$, and the sub-mm - mm wavelength range. The hottest dust located in the inner disk emits in the $1-10 \mu \mathrm{m}$ wavelength range. Dust cannot survive temperatures above $\sim 1500 \mathrm{~K}$ (with one or two exceptions, e.g. $\mathrm{Al}_{2} \mathrm{O}_{3}$ ). Indeed, most young stars show a near-IR excess above the photospheric emission due to dust at roughly that temperature, and interferometric observations of Herbig Ae/Be stars confirm that the distance at which the hottest dust is located is consistent with temperatures in the range $1000-1500 \mathrm{~K}$. Furthermore, the distance of the dust scales as $r \propto L^{1 / 2}$, where $\mathrm{L}$ is the luminosity of the star (Monnier et al. 2005). Recently this relation has also been found for T Tau stars (Anthonioz et al. 2014), however, more extended near-IR emission from scattered light was also found for some of these stars. The interferometric observations of Herbig Ae/Be stars and T Tau stars show that there is an innermost, dust-free cavity in disks. Hot dust represents only a very small fraction $\left(\approx 10^{-6}\right)$ of the total dust mass.

Herbig Ae/Be stars show a remarkably large near-IR excess, that can take up as much as $25 \%$ of the total disk luminosity (Natta et al. 2001). This has been interpreted as due to the presence of a puffed-up inner rim caused by the direct irradiation of the inner rim of the disk by the star. Radiative transfer models indeed show an increased scale-height of the innermost disk regions (Dullemond et al. 2001), although these models have a difficulty in accounting for the observed near-IR excess. An (additional) optically thin dust halo is introduced to account for the observed near-IR emission (Vinković et al. 2006).

Warm (100-500 K) dust, located in the disk surface layers from 3-5 to a few tens of AU (distances of course depend on the luminosity of the central star!), dominate in the 10-100 $\mu \mathrm{m}$ range. Typically 
$10 \%$ of the mass is in the warm dust component. The $10-100 \mu \mathrm{m}$ spectral range contains many vibrational resonances of dust species, and so the mineralogy of this warm dust can be very well studied. If the disk is not viewed edge-on, these resonances are seen in emission. This is because of the vertical temperature gradient in the irradiated disk, with a increasing temperature when going to the disk surface. Interestingly, settling timescales for small dust grains at these distances are of the order of $10^{5}$ years or less, much shorter than the disk lifetime. Therefore one would not expect to see the signature of small $(1 \mu \mathrm{m})$ grains in the disk surface. Such grains must therefore be constantly supplied to the disk surface, due to turbulent mixing from the disk mid-plane. Indeed, at any time the population of grains in the disk is a delicate balance between growth, destruction, and mixing.

At sub-mm wavelengths, the cold dust grains in the disk mid-plane dominate the emission. Typically $90 \%$ of the dust mass is in this cold dust component. This is because the bulk of the dust mass in disks has settled to the mid-plane, and despite its low $(<50 \mathrm{~K})$ temperature, it still dominates the disk emission at (sub-) millimeter wavelengths. When the disk is optically thin the slope of the SED at sub-millimeter and millimeter wavelength ranges can be used to probe (limits on) the size distribution of the cold mid-plane grains (e.g. Testi et al. 2003). One can assume the Rayleigh-Jeans approximation for the source function (Planck function $\mathrm{B}_{v}$ ) of the grains, so that the flux:

$$
F_{v} \propto B_{v}\left(T_{\text {dust }}\right) \kappa_{v}^{\mathrm{abs}} \propto v^{2} \kappa_{v}^{\mathrm{abs}} \propto v^{2+p}
$$

In interstellar space, the slope of the millimeter continuum emitted by cold dust is $F_{v} \propto v^{\alpha}$ with $\alpha \approx 3.7$, indicative of very small grains in the Rayleigh limit (Testi et al. 2014). This is very different for protoplanetary disks. Spectral slopes at (sub-) millimeter wavelengths have been measured now for large samples of T Tau stars and Herbig Ae/Be stars, and generally values of $\alpha$ significantly smaller than the ISM value have been derived, even as small as $\alpha=2$, corresponding to "black body" grains with a wavelength independent opacity (Testi et al. 2014, and references therein). This implies that the emission is dominated by large grains (size parameter $x>1$ ), typically larger than $\approx 1 \mathrm{~mm}$. In some disks even the radio emission at $\approx 1 \mathrm{~cm}$ is dominated by cold dust, and limits on grain size as high as $1 \mathrm{~cm}$ can be derived. Such observations confirm the presence of a reservoir of large cold mid-plane grains, and larger particles can of course not be excluded. When spatially resolved images at different $\mathrm{mm}$ wavelengths are available, the spectral slope can be studied as a function of distance. Since grain settling timescales are short near the star, grain growth is expected to have progressed further in the inner disk, and so the millimeter spectral slope should increase outwards. This has indeed been observed in a few cases (Pérez et al. 2012; Testi et al. 2014).

The mass of cold dust in the disk $\mathbf{M}_{\text {dust }}$ can be estimated in a simple way, assuming the dust is optically thin, the Rayleigh-Jeans approximation applies, and the dust grains are small compared to the wavelength:

$$
F_{v}=\frac{\pi B_{v}\left(T_{\text {dust }}\right) \kappa_{v}^{\text {abs }} M_{\text {dust }}}{D^{2}}
$$

where $D$ is the distance to the disk, and $F_{v}$ is the observed flux (in frequency units).

\subsection{Disk geometry and SED shape}

The SEDs of protoplanetary disks are sensitive to disk geometry, but there are many degeneracies, see chapter by Woitke (2015). Depending on the adopted dust properties, a range of disk geometries can fit an observed SED. Clearly direct imaging provides the strongest constraints on disk geometry, but nevertheless SEDs do contain some information. In Fig. 5 some examples of disk geometries and corresponding SED shapes are given. A lack of near-IR excess is an indication for dust depletion 

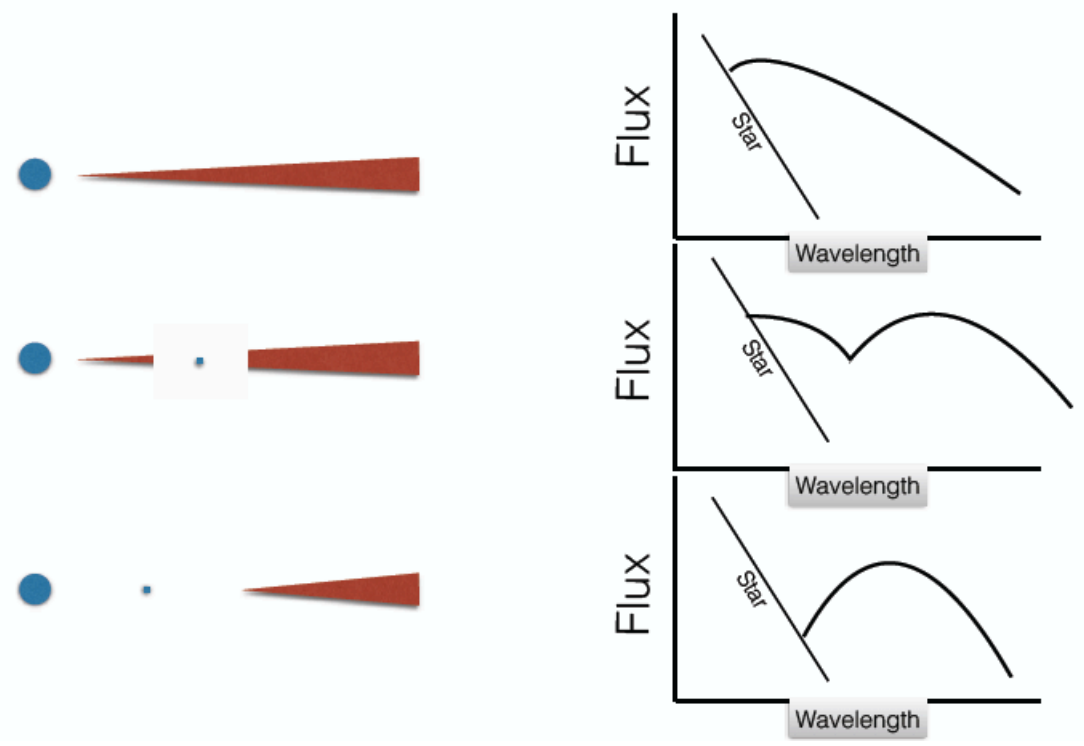

Figure 5. Disk geometries and corresponding schematic SED shapes. From top to bottom: a continuous disk, a disk with a gap formed by a young planet, and a disk with a large hole, with only an outer disk still present. The disk gap causes a dip in the SED at the wavelength corresponding to the temperature that grains at the gap distance would have. The disk with large inner hole shows no near-IR excess.

in the innermost disk regions: the hottest dust is observed to be (much) cooler than the dust sublimation temperature: an inner hole exists. These disks are classified as transitional: disk dissipation is on-going, possibly due to the formation of planets that clear part of the disk (e.g. Calvet et al. 2002; Sicilia-Aguilar et al. 2006). Note that transitional disks can still have a (weak) near-IR excess, indicative of some hot dust left in the innermost disk regions.

Disk gaps are regions in the disk where the surface density of dust and gas is significantly lower than in a continuous disk. These gaps are more difficult to detect in SEDs, especially if the disk gap is small compared to the size of the disk. Disk gaps remove emission from dust grains at wavelengths corresponding to the temperature of the removed grains. For Herbig Ae/Be stars, based on the SED shape, Meeus et al. (2001) defined two groups, with group I SEDs having an increasing flux towards the mid-IR, while the group II disks show a more steeply declining flux with wavelength. The group I SEDs were originally interpreted in the context of flaring disk geometries (Dullemond \& Dominik 2004), but more recently it has been recognized that group I SEDs are in fact transitional disks with large disk gaps, in the distance range of several tens of AU (Maaskant et al. 2013). The sharp rise of flux in the $10-30 \mu \mathrm{m}$ spectral region is due to the inner wall of the outer disk, that is directly irradiated by the star. The Group II Herbig Ae/Be star SEDs are self-shadowed: the outer disk is hidden in the shadow cast by the puffed-up inner rim (Dullemond \& Dominik 2004). The evolutionary link between the group I and group II SEDs is still unclear. A similar discussion about the SED evolution of disks surrounding T Tau stars is presented in Currie (2010). 
HD100546 on OD 211 Fully calibrated SED

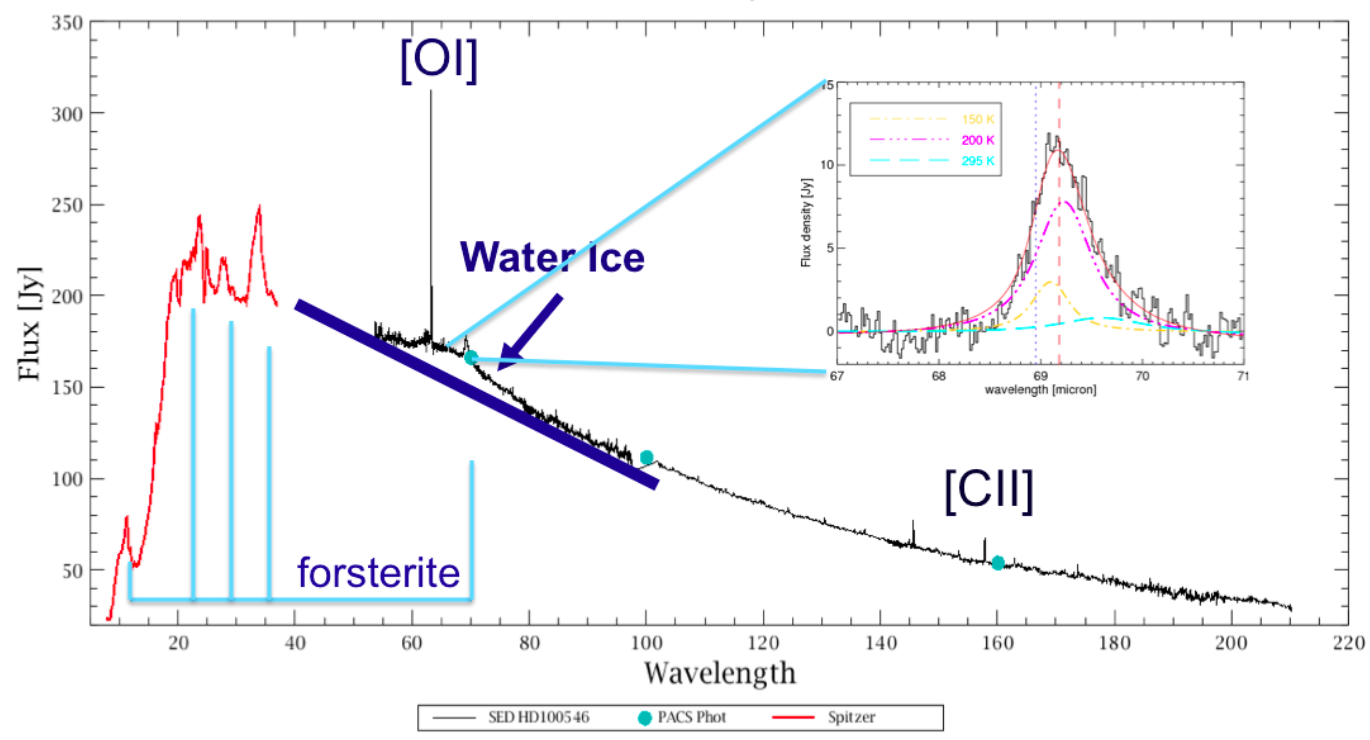

Figure 6. Observed infrared spectrum of the Herbig Be star HD 100546 (Figure courtesy of J. Bouwman; see also Waters et al., 2015 (in press); Bouwman et al., in preparation). Shown are the Spitzer and Herschel data, as well as the positions of the strongest forsterite bands. The inset shows the $69 \mu \mathrm{m}$ forsterite band, and model fits assuming different temperatures of the grains.

\subsection{Spectroscopy of dust}

A wealth of infrared spectra of disks around young stars has been obtained using the Infrared Space Observatory, the Spitzer Space Telescope and the Herschel Space Observatory (e.g. Juhász et al. 2010b; Meeus et al. 2001; Olofsson et al. 2009; Sicilia-Aguilar et al. 2007; Watson et al. 2009). As an example the infrared spectrum of the Herbig Be star HD100546 is shown in Fig. 6. Very prominent emission bands of small dust grains in the disk atmosphere (Bouwman et al., in preparation) dominate the spectrum. Species identified are forsterite $\left(\mathrm{Mg}_{2} \mathrm{SiO}_{4}\right)$, which is the $\mathrm{Mg}$-rich end member of the olivines. The spectrum also shows clear emission from crystalline water ice (near $60 \mu \mathrm{m}$ ). The 69 $\mu \mathrm{m}$ band of forsterite, detected using Herschel (Sturm et al. 2010), is very sensitive to the Fe/Mg ratio in the solid. In addition, the wavelength and shape are also temperature sensitive (Suto et al. 2006). The band shifts to longer wavelengths as more Fe is added to the lattice, and the observations are consistent with an $\mathrm{Fe}$ content of less than $1 \%$. The abundance of forsterite in disks is usually only a few per cent, but in some temperature ranges it can be the most abundant silicate species. This is especially true in the innermost disk regions, where temperatures above the glass temperature of silicates $(\approx 1000 \mathrm{~K})$ prevail. The innermost disk regions must be fully crystalline. Interferometric observations of Herbig Ae/Be disks confirm that the inner disks are highly crystalline, up to $100 \%$ (van Boekel et al. 2004). Spectroscopic surveys have also revealed the presence of enstatite $\left(\mathrm{MgSiO}_{3}\right)$ and of Silica $\left(\mathrm{SiO}_{2}\right)$ (Juhász et al. 2010a).

The spectrum of HD100546, and many other spectra of Herbig Ae/Be and T Tau disks, shows that also much colder crystalline silicates are present, that must be located at much larger distance from the star. Since crystalline grains are not present in the ISM, the cold crystals must have been produced 
in the disk. This can be done through transport of grains from the inner disk to the outer disk. This transport can be either through radial mixing in the disk mid-plane, or transport of grains entrained in a disk wind/outflow. An alternative to transport of grains from the inner to the outer disk are local processes that heat up grains to temperatures above the glass temperature, such as lightning. Direct evidence for the production of crystalline silicates and transport to the outer disk regions has come from observations of the outburst of EX Lupi (Abraham et al. 2009), during which crystalline silicates were formed. Subsequent monitoring of the produced crystalline silicates with Spitzer showed a rapid outward transport of the crystals (Juhász et al. 2012). Chemical equilibrium dust condensation and radial mixing models predict that at the highest temperatures forsterite condenses, and that at lower temperatures a conversion to enstatite occurs (Gail 2004). Observations show that an opposite trend is observed, i.e. enstatite is more concentrated in the inner disk, while forsterite is more concentrated in the outer disk regions (Juhász et al. 2010b). In the particular case of HD100546, the forsterite grains are located in the inner wall of the outer disk, at $\sim 13 \mathrm{AU}$ distance (Mulders et al. 2011). It is possible that a low mass companion in the disk gap causes a collisional cascade, producing smaller grains near the inner edge of the outer disk. It is likely that no single mechanism is responsible for the spatial distribution of crystalline silicates in disks, but that several contribute at any time during disk evolution.

\section{Imaging of protoplanetary disks}

Direct imaging of protoplanetary disks shows a bewildering richness of structures that are the imprint of planet formation and disk dissipation on disk structure. Images taken at different wavelengths probe different dust components. Disks can show a remarkable difference in geometry when imaged at different wavelengths. This proves that different kinds of dust species (small grains, large grains) can have different spatial distributions. In turn, the spatial distribution of gas and dust in disks can be very different: examples are known of dust gaps in disks that still have substantial amounts of gas (e.g. Casassus et al. 2014; Maaskant et al. 2014). The images show evidence for disk gaps, holes, spiral density waves and dust traps.

Images of protoplanetary disks at short wavelengths usually show starlight reflected by the dust in the disk surface layers. When the disk is viewed edge-on against a bright background emission, it can be detected as a "silhouette" in absorption. Many protoplanetary disks have been detected in scattered light at optical and near-IR wavelengths. The scattered light images probe the disk surface with is in direct view of the star, and therefore originates from the disk surface layers (starlight does not penetrate deeply into the disk). The spatial and wavelength dependence of the intensity distribution can be used to derive information about the surface density distribution of the gas disk (using models), and about the size distribution of the grains and their albedo. The scattered light is dominated by small grains (a few microns or less) in the disk surface layers, that have not (yet) settled to the mid-plane. Because scattered light images of disks are always taken with a coronograph, obscuring the inner 10 - $20 \mathrm{AU}$ of the disk, not much is known of the scattering properties of grains in the innermost disk regions. Interferometry may help here. Recently a sample of T Tau stars was studied using the VLTI, and it was shown that the spatial scale of the emission can only be understood if the grains are scattering (Green et al. 2013).

Many disk images show evidence for spiral density waves (e.g. Hashimoto et al. 2011; Muto et al. 2012). These can be the result of the interaction between the disk and a low mass companion (located in a disk gap), but also due to gravitational instability in a massive disk.

Infrared and millimeter imaging provides information about the spatial distribution of the warm and cold grains, respectively. Millimeter interferometers such as the SMA and in particular ALMA 


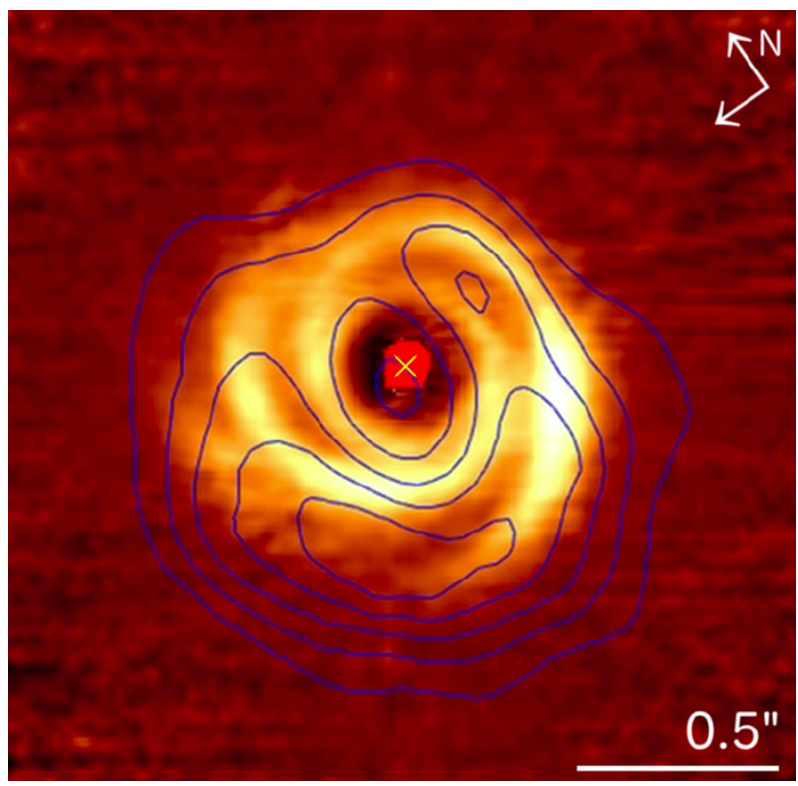

Figure 7. Near infrared $\left(\mathrm{K}_{s}\right)$ polarized scattered light image of the protoplanetary disk surrounding the young star HD 135344B (Garufi et al. 2013, reproduced with permission (c) ESO). Notice the prominent spiral density wave structure in scattered light. Contours show the sub-mm wavelength continuum emission taken with the SMA. The inner radius of the gap in scattered light (tracing small grains) is significantly smaller than that seen at sub-mm wavelengths (tracing large grains).

at (sub-) millimeter are providing a first glance on the sub-arcsecond distribution of the large grains in disks (e.g. Andrews et al. 2011). These observations for instance show that the size of the disk in HD100546 measured in the sub-mm continuum is much smaller than the disk detected in scattered light (Pineda et al. 2014). This can be understood in the context of the inward drift of large, millimetersized dust particles in the disk mid-plane. Smaller grains, present in the disk surface layers, are better coupled to the gas and do not show this inward drift. This can explain the difference in spatial distribution of large and small grains.

Small and large dust grains can also show a different spatial distribution near the edge of disk gaps. A beautiful example is shown in figure 7 for HD 135344B (Garufi et al. 2013). Despite the difference in angular resolution between the near-IR and sub-mm images, it is clear that the outer radius of the disk gap detected in polarized scattered light (tracing small grains) is significantly smaller than that imaged in the sub-mm and millimeter (tracing large, mm-sized grains). This difference may be due the detailed gas density structure of the outer disk near the edge of the gap, resulting from the interaction between a planet in the gap and the gas in the outer disk. This results in a pressure bump which can be at a larger distance than the edge of the gas gap. Large grains tend to pile up in a pressure maximum, while small grains are well coupled to the gas and therefore trace the gas distribution.

ALMA has also revealed the presence of dust traps (Fukagawa et al. 2013; van der Marel et al. 2013) in transitional disks: radially and azimuthally confined concentrations of large dust grains. These dust traps can be explained by radial and azimuthal pressure bumps in the disk near the edge of the gap, caused by the presence of a low mass companion inside the gap, possibly near the edge of the gap. Because large dust grains always drift in the direction of a pressure maximum, an accumulation 
of large grains in a narrow region of the disk can occur. Smaller grains are much better coupled to the gas, and so show a much less pronounced asymmetry in the dust distribution.

Perhaps the most exiting recent result of disk imaging is the detection of small objects in disk gaps, whose properties are consistent with protoplanets (e.g. Currie et al. 2014; Quanz et al. 2014; Reggiani et al. 2014). These objects can provide a direct link between protoplanetary disks and mature planetary systems.

\section{Concluding remark}

These lecture notes are by necessity incomplete in covering the literature, and the cited papers are meant as examples of the observations described.

Acknowledgements The research leading to these results has received funding from the European Union Seventh Framework Programme FP7-2011 under grant agreement no 284405.

\section{References}

Abraham, P., Juhász, A., Dullemond, C. P., et al. 2009, Nature, 459, 224

Andrews, S. M., Wilner, D. J., Espaillat, C., et al. 2011, The Astrophysical Journal, 732, 42

Anthonioz, F., Ménard, F., Pinte, C., et al. 2014, arXiv.org

Calvet, N., D’Alessio, P., Hartmann, L., et al. 2002, The Astrophysical Journal, 568, 1008

Casassus, S., van der Plas, G., M, S. P., et al. 2014, Nature, 493, 191

Costantini, E., de Vries, C., \& Zeegers, S. 2014, The X-ray Universe 2014, 54

Costantini, E., Pinto, C., Kaastra, J. S., et al. 2012, Astronomy \& Astrophysics, 539, 32

Currie, T. 2010, arXiv.org

Currie, T., Muto, T., Kudo, T., et al. 2014, The Astrophysical Journal Letters, 796, L30

Draine, B. T. 2004, Astrophysics of Dust in Cold Clouds (Springer-Verlag Berlin Heidelberg, ISBN 978-3-540-40838-3), 213

Dullemond, C. P. \& Dominik, C. 2004, A\&A, 417, 159

Dullemond, C. P., Dominik, C., \& Natta, A. 2001, The Astrophysical Journal, 560, 957

Dullemond, C. P., Hollenbach, D., Kamp, I., \& D’Alessio, P. 2007, Protostars and Planets V, 555

Fukagawa, M., Tsukagoshi, T., Momose, M., et al. 2013, Publications of the Astronomical Society of Japan, 65, L14

Gail, H. P. 2004, Astronomy \& Astrophysics, 413, 571

Garufi, A., Quanz, S. P., Avenhaus, H., et al. 2013, A\&A, 560, A105

Green, J. D., Evans, II, N. J., Jørgensen, J. K., et al. 2013, ApJ, 770, 123

Hashimoto, J., Tamura, M., Muto, T., et al. 2011, The Astrophysical Journal Letters, 729, L17 
Summer School "Protoplanetary Disks: Theory and Modeling Meet Observations"

Juhász, A., Bouwman, J., Henning, T., et al. 2010a, The Astrophysical Journal, 721, 431

Juhász, A., Bouwman, J., Henning, T., et al. 2010b, arXiv.org, 721, 431

Juhász, A., Dullemond, C. P., van Boekel, R., et al. 2012, The Astrophysical Journal, 744, 118

Lee, J. C. \& Ravel, B. 2005, The Astrophysical Journal, 622, 970

Maaskant, K. M., Honda, M., Waters, L. B. F. M., et al. 2013, Astronomy \& Astrophysics, 555, 64

Maaskant, K. M., Min, M., Waters, L. B. F. M., \& Tielens, A. G. G. M. 2014, Astronomy \& Astrophysics, 563, 78

Meeus, G., Waters, L. B. F. M., Bouwman, J., et al. 2001, Astronomy \& Astrophysics, 365, 476

Monnier, J. D., Millan-Gabet, R., Billmeier, R., et al. 2005, The Astrophysical Journal, 624, 832

Mulders, G. D., Waters, L. B. F. M., Dominik, C., et al. 2011, Astronomy \& Astrophysics, 531, 93

Muto, T., Grady, C. A., Hashimoto, J., et al. 2012, The Astrophysical Journal Letters, 748, L22

Natta, A., Prusti, T., Neri, R., et al. 2001, Astronomy \& Astrophysics, 371, 186

Olofsson, J., Augereau, J. C., van Dishoeck, E. F., et al. 2009, Astronomy \& Astrophysics, 507, 327

Pérez, L. M., Carpenter, J. M., Chandler, C. J., et al. 2012, The Astrophysical Journal Letters, 760, L17

Pineda, J. E., Quanz, S. P., Meru, F., et al. 2014, The Astrophysical Journal Letters, 788, L34

Quanz, S. P., Amara, A., Meyer, M. R., et al. 2014, arXiv.org

Reggiani, M., Quanz, S. P., Meyer, M. R., et al. 2014, The Astrophysical Journal Letters, 792, L23

Sicilia-Aguilar, A., Hartmann, L., Calvet, N., et al. 2006, The Astrophysical Journal, 638, 897

Sicilia-Aguilar, A., Hartmann, L. W., Watson, D., et al. 2007, The Astrophysical Journal, 659, 1637

Sturm, B., Bouwman, J., Henning, T., et al. 2010, A\&A, 518, L129

Suto, H., Sogawa, H., Tachibana, S., et al. 2006, Monthly Notices of the Royal Astronomical Society, 370,1599

Testi, L., Birnstiel, T., Ricci, L., et al. 2014, Protostars and Planets VI, 339

Testi, L., Natta, A., Shepherd, D. S., \& Wilner, D. J. 2003, A\&A, 403, 323

van Boekel, R., Min, M., Leinert, C., et al. 2004, Nature, 432, 479

van der Marel, N., van Dishoeck, E. F., Bruderer, S., et al. 2013, Science, 340, 1199

Vinković, D., Ivezić, Ž., Jurkić, T., \& Elitzur, M. 2006, The Astrophysical Journal, 636, 348

Watson, D. M., Leisenring, J. M., Furlan, E., et al. 2009, arXiv.org, 180, 84

Woitke, P. 2015, in EPJ Web of Conferences, Vol. 102, Summer School on Protoplanetary Disks:

Theory and Modeling Meet Observations, ed. I. Kamp, P. Woitke, \& J. D. Ilee 
